\title{
The effect of enhanced bone marrow in conjunction with 3D-printed PLA-HA in the repair of critical-sized bone defects in a rabbit model
}

\author{
Zhiqing Liu ${ }^{1,2 \#}$, Wenxiang Chu ${ }^{1,3 \#}$, Linyuan Zhang ${ }^{1}$, Yueting Wang ${ }^{1}$, Zanjing Zhai ${ }^{1}$, Fengxiang Liu ${ }^{1}$ \\ ${ }^{1}$ Department of Orthopedics, Shanghai Ninth People's Hospital, Shanghai Jiao Tong University School of Medicine, Shanghai, China; ${ }^{2}$ Department \\ of Orthopedic Surgery, Tongji Hospital, Tongji University School of Medicine, Shanghai, China; ${ }^{3}$ Department of Orthopedic Surgery, Changzheng \\ Hospital, Second Military Medical University, Shanghai, China \\ Contributions: (I) Conception and design: F Liu, Z Liu; (II) Administrative support: F Liu, Z Zhai; (III) Provision of study materials or patients: W \\ Chu, L Zhang, Y Wang; (IV) Collection and assembly of data: Z Liu, W Chu; (V) Data analysis and interpretation: Z Liu, Z Zhai; (VI) Manuscript \\ writing: All authors; (VII) Final approval of manuscript: All authors. \\ \#These authors contributed equally to this work. \\ Correspondence to: Zanjing Zhai; Fengxiang Liu. Department of Orthopedics, Shanghai Ninth People's Hospital, Shanghai Jiao Tong University \\ School of Medicine, Shanghai, China. Email: zanjing_zhai@163.com; liu_fengxiang@126.com.
}

Background: Traditionally, the iliac crest has been the most common harvesting site for autologous bone grafts; however, it has some limitations, including poor bone availability and donor-site morbidity. This study sought to explore the effect of enhanced bone marrow (eBM) in conjunction with three-dimensional (3D)printed polylactide-hydroxyapatite (PLA-HA) scaffolds in the repair of critical-sized bone defects in a rabbit model.

Methods: First, 3D-printed PLA-HA scaffolds were fabricated and evaluated using micro-computed tomography $(\mu \mathrm{CT})$ and scanning electron microscopy (SEM). Twenty-seven New Zealand white rabbits were randomly divided into 3 groups ( $\mathrm{n}=9$ per group), and the defects were treated using 3D-printed PLAHA scaffolds (the PLA-HA group) or eBM in conjunction with 3D-printed PLA-HA scaffolds (the PLAHA/eBM group), or were left untreated (the control group). Radiographic, $\mu \mathrm{CT}$, and histological analyses were performed to evaluate bone regeneration in the different groups.

Results: The 3D-printed PLA-HA scaffolds were cylindrical, and had a mean pore size of $500 \pm 47.1 \mu \mathrm{m}$ and $60 \% \pm 3.5 \%$ porosity. At 4 and 8 weeks, the lane-sandhu X-ray score in the PLA-HA/eBM group was significantly higher than that in the PLA-HA group and the control group $(\mathrm{P}<0.01)$. At 8 weeks, the $\mu C T$ analysis showed that the bone volume (BV) and bone volume/tissue volume (BV/TV) in the PLA-HA/ eBM group were significantly higher than those in the PLA-HA group and the control group $(\mathrm{P}<0.01)$. Hematoxylin and eosin staining indicated that the new bone area in the PLA-HA/eBM group was significantly higher than that in the PLA-HA group and the control group $(\mathrm{P}<0.01)$.

Conclusions: The group that was treated with eBM in conjunction with 3D-printed PLA-HA showed enhanced bone repair compared to the other 2 groups. PLA-HA/eBM scaffolds represent a promising way to treat critical-sized bone defects.

Keywords: Critical-sized bone defects; enhanced bone marrow (eBM); mesenchymal stem cells (MSCs); 3D-printing technique; PLA-HA

Submitted Dec 28, 2020. Accepted for publication May 16, 2021.

doi: 10.21037/atm-20-8198

View this article at: https://dx.doi.org/10.21037/atm-20-8198 


\section{Introduction}

The treatment of critical-sized bone defects caused by high-energy trauma, infection, or tumor resection remains a substantial challenge in the field of orthopedics (1-3). Traditionally, the iliac crest has been the most common harvesting site for autologous bone grafts; however, it has some limitations, including poor availability and donor-site morbidity (4-6). Therefore, new bone substitutes need to be developed for the repair of large bone defects.

Intramedullary reaming and nailing is widely known to be suitable for treating long-bone nonunions (7). In the process of reaming, debris, which is referred to as enhanced bone marrow (eBM), is produced. Previous studies have shown that eBM obtained from the reaming of long-bone medullary canals containing large quantities of stem cells has the potential to accelerate bone repair and regeneration (8-10). Given its excellent properties for bone repair, eBM has become an alternative autologous bone graft harvested from bone cavity and overcomes some of the drawbacks of iliac crest bone grafts (10). However, eBM does not possess the gross anatomical structure required to guide bone repair and regeneration.

Recently, the field of bone tissue engineering (BTE) has been developing rapidly, and various promising biomaterials have emerged and have been widely applied for bone repair and regeneration $(11,12)$. The use of osteogenic cells combined with various growth factors and biocompatible scaffolds can greatly promote bone repair. As one of the 3 key elements of BTE, scaffolds can provide the necessary spatial and structural support for cellular growth. An ideal tissue engineering scaffold should exhibit some excellent properties, such as biocompatibility, osteoinductivity, and osteoconductivity, and possess an appropriate pore size and porosity $(13,14)$.

In recent decades, hydroxyapatite [HA; $\left.\mathrm{Ca}_{5}\left(\mathrm{PO}_{4}\right)_{3} \mathrm{OH}\right]$ and beta-tricalcium phosphate [ $\beta$-TCP; $\left.\mathrm{Ca}_{3}\left(\mathrm{PO}_{4}\right)_{2}\right]$ have been widely applied to facilitate bone repair and regeneration in clinical settings due to their recognized biocompatibility and osteoconductivity $(15,16)$. Previous studies have also shown that single-phase materials cannot perfectly meet the needs of BTE scaffolds (17). To construct synthetic scaffolds, HA and $\beta$-TCP have been incorporated into polymers, such as poly(lactic acid) (PLA), polycaprolactone (PCL), poly(ethylene glycol) (PEG), and poly(lactic-co-glycolic acid) (PLGA) (18). The polylactide-hydroxyapatite (PLA-HA) composite has great biocompatibility, degradability and good mechanical properties, and thus has attracted significant attention and been investigated widely (19-22). However, the osteoinductivity of the scaffold needs to be enhanced. Thus, we hypothesized that the PLA-HA composite could provide mechanical support for seeded eBM and be used to construct a complex scaffold to facilitate bone repair and regeneration.

Three-dimensional (3D)-printing techniques represent a new way to construct BTE scaffolds. These techniques can be applied to fabricate different forms on the basis of bone defects, and provide a suitable environment for cell attachment and proliferation $(23,24)$. They have been used for the formation of polymer products, including implants and medical devices. In the present study, we applied a 3D-printing technique to build synthetic PLA-HA scaffolds.

In the present study, we used a method (25) that mimicked the reamer-irrigator-aspirator technique to acquire eBM. Using a 3D-printing technique, 3D-printed PLA-HA scaffolds were successfully fabricated. We hypothesized that eBM in conjunction with PLA-HA could be used to facilitate bone repair and bone regeneration in the treatment of critical-sized bone defects. We present the following article in accordance with the ARRIVE reporting checklist (available at https://dx.doi.org/10.21037/atm-20-8198).

\section{Methods}

\section{Preparation and characterization of $3 D$-printed PLA-HA bybrid scaffolds and cell seeding onto scaffolds}

All chemicals were obtained from Sigma Aldrich (Shanghai, China). A 3D-Bioplotter ${ }^{\mathrm{TM}}$ (Envision TEC GmbH, Germany) was used to construct the 3D-printed scaffolds using a computer. First, PLA particles (mean molecular weight $\sim 20.0 \times 10^{4} \mathrm{Da}$ ) were dissolved in 1, 4-dioxane. HA powders (particle size $\sim 50 \mathrm{~m}$ ) were then added to the PLA solution and stirred for $12 \mathrm{~h}$ to form a uniform PLA/HA paste using a ratio of 7:3. Next, the cylinder models were loaded into $3 \mathrm{D}$ bioplotter software. The $3 \mathrm{D}$-printed scaffolds were fabricated to have an outer diameter of $5 \mathrm{~mm}$, an inner diameter of $2 \mathrm{~mm}$, and a height of $15 \mathrm{~mm}$ for the in-vivo experiments.

High-resolution micro-computed tomography (Scanco Medical, $\mu$ CT; Switzerland) was used to assess the porosity and average pore size of the scaffolds. The data were analyzed by $\mu C T$. Scanning electron microscopy (SEM; USA) was performed to observe the microstructure and morphology of the 3D-printed scaffolds. 


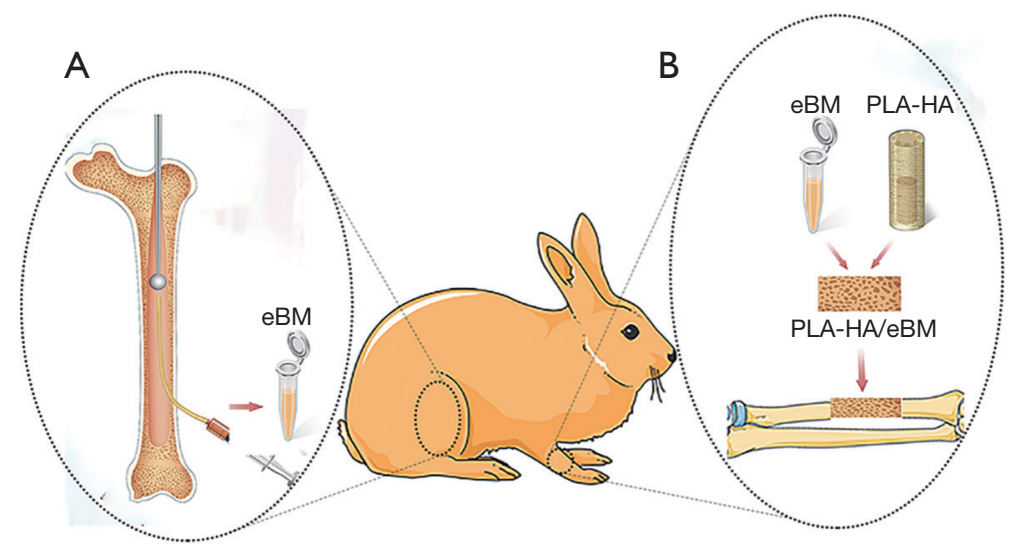

Figure 1 Schematic illustration of enhanced bone marrow (eBM) in conjunction with three-dimensional (3D) PLA-HA scaffolds on repairing long bone defects in a rabbit model. (A) eBM harvest. (B) PLA-HA/eBM scaffolds were implanted into bone defects.

Mesenchymal stem cells (MSCs) isolated from the eBM were used. Cell suspension was seeded at a density of $1 \times 10^{5}$ cells on the $3 \mathrm{D}$-printed scaffolds in 24 -well plates. Cell spreading and focal adhesion formation were assessed after 24 hours of culture $\left(37^{\circ} \mathrm{C}\right.$ and $5 \%$ carbon dioxide in a humidified incubator). SEM was used to observe the attachment of cells onto the scaffolds.

\section{Animal groups}

Twenty-seven adult New Zealand white rabbits were included (aged 6 months and weighing $2.5 \pm 0.25 \mathrm{~kg}$ ). The rabbits were housed in separate cages, fed a standard diet, and allowed free movement during the experimental period. The 27 rabbits were randomly divided into the following 3 groups (n=9): (I) the control group (critical-sized bone defects without treatment); (II) the PLA-HA group (critical-sized bone defects treated with implantation of 3D-printed PLA-HA scaffolds); or (III) the PLA-HA/eBM group (critical-sized bone defects treated with $3 \mathrm{D}$-printed PLA-HA scaffolds in conjunction with eBM). All experiments were performed under a project license (no. HKDL [2018] 499) granted by the Animal Care Committee of Shanghai Jiao Tong University, in compliance with the guidelines for the care and use of animals of Shanghai Ninth People's Hospital Affiliated Shanghai Jiao Tong University School of Medicine.

\section{Surgical procedure}

A large bone defects rabbit model was used in this study. Anesthesia was given in the form of $3 \%$ pentobarbital sodium $(30 \mathrm{mg} / \mathrm{kg})$ via intramuscular injection. Unilateral 15 -mm critical-sized defects were constructed in the middle of the left radius. In the control group, no treatment was given. In the PLA-HA group, a 3D-printed PLA-HA scaffold was inserted at each bone defect site. In the PLAHA/eBM group, a 2-mm drill was used to ream the distal femur and a medullo-puncture needle was used in the proximal femur. Suction was carried out simultaneously with drill reaming. A $10-\mathrm{mL}$ syringe was used to inject eBM into the hollow tunnel of the scaffold. Subsequently, the scaffold material in the tube was soaked with the eBM and mixed well for 10 minutes. The 3D-printed PLA-HA scaffold together with the eBM was inserted into the bone defect site. Figure 1 provides a schematic illustration of the use of eBM in conjunction with 3D-printed PLA-HA scaffold in the repair of a critical-sized bone defect in the rabbit model. X-ray and $\mu \mathrm{CT}$ evaluation of critical-sized bone defect repair.

At 0 weeks (i.e., immediately after the operation), 4 and 8 weeks, $\mathrm{X}$-rays of the defect sites were performed using an X-ray machine (Faxitron X-ray Corporation, USA). Lane-sandhu X-ray scores (26) were used to compare the effect of bone healing in each group. At 8 weeks, all of the animals were sacrificed, and a $\mu \mathrm{CT}$ ( $\mu \mathrm{CT}-80$, Switzerland) analysis was subsequently conducted to assess bone healing. A global threshold procedure was used to analyze the bony compartments. The threshold of bony tissue was greater than or equal to 150 . The threshold of soft tissue and implanted composite scaffolds was below 150. In this study, a cylindrical area of $15 \mathrm{~mm}$, including the bone defect site of the radius in the center, was selected as the region of interest to quantify the bone volume (BV) and the bone 

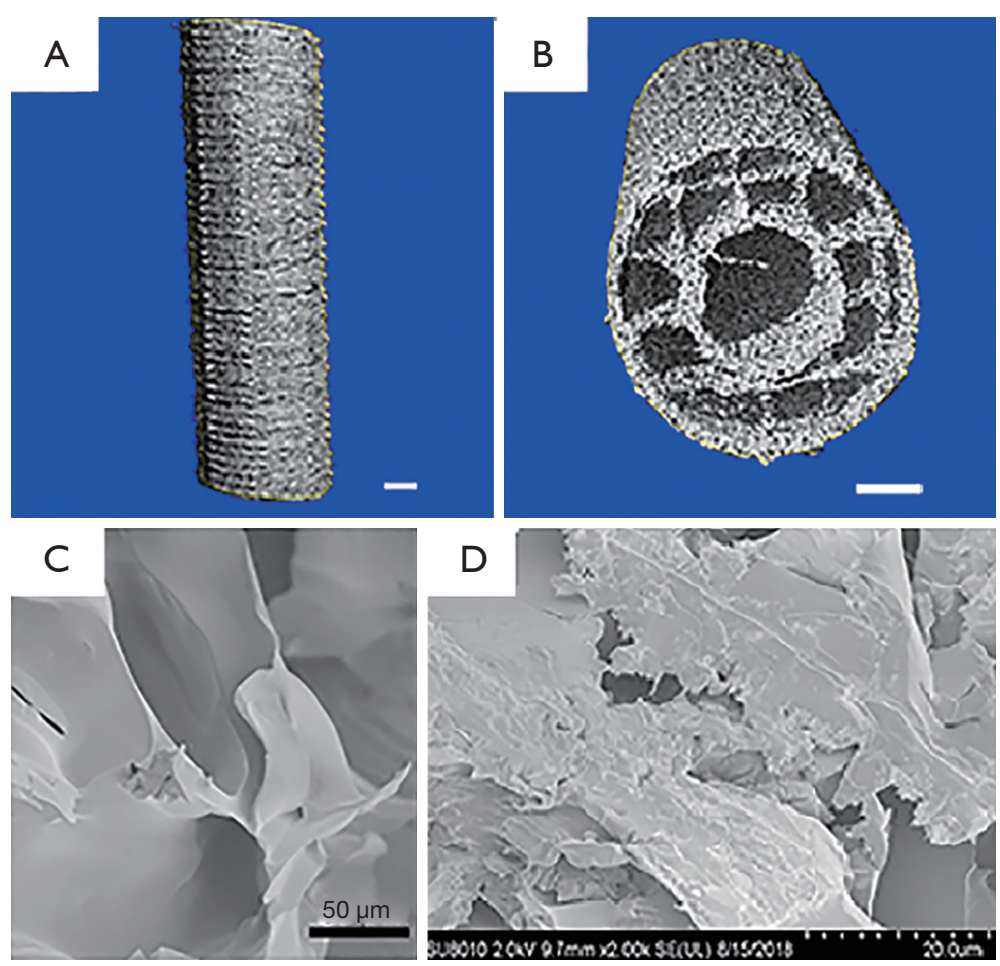

Figure 2 Microstructure and morphology of 3D PLA-HA scaffolds. (A,B) $\mu$ CT (scar bar =1 mm), (C) Scanning electron microscopy (SEM) of 3D PLA-HA scaffolds. (D) Well spread MSCs was observed on the scaffold (scar bar =20 $\mu \mathrm{m}$ ).

volume/total volume (BV/TV).

\section{Histological evaluation}

After $\mu \mathrm{CT}$ scanning, the radius specimens were decalcified in $12 \%$ ethylenediaminetetraacetic acid disodium salt (EDTA-2Na; Sangon Biotech, China) for 4-5 weeks, dehydrated in a graded series of alcohol, and embedded in paraffin for histological sectioning. Sections with a thickness of $6 \mu \mathrm{m}$ were cut and stained with hematoxylin and eosin (HE). The stained sections were observed microscopically, and newly formed bone was evaluated with Image Pro Plus 6.0 software (Media Cybernetics, USA).

\section{Statistical analysis}

One-way analysis of variance using SPSS 22.0 software (SPSS Inc., Chicago, IL, USA) was conducted to analyze the results. The results were expressed as the mean \pm standard deviation (SD) from at least 3 independent experiments. A value of $\mathrm{P}<0.05$ was defined as being statistically significant.

\section{Results}

\section{Structure of the 3D-printed PLA-HA scaffolds and cell attachment on the scaffolds}

We successfully fabricated 3D-printed PLA-HA scaffolds using a $3 \mathrm{D}$-printing technique. The 3D-printed PLA-HA scaffolds were indicated by $\mu \mathrm{CT}$ and SEM to be hollow cylindrical structures, similar to long bones, possessing a porous wall structure, with a pore size of $500 \pm 47.1 \mu \mathrm{m}$ and $60 \% \pm 3.5 \%$ porosity (Figure 2 ). Well-spread MSCs were observed on the scaffolds.

\section{Gross observation, $X$-ray, and $\mu C T$ evaluation}

All animals recovered well from the operative procedure (Figure 3). No infections or other complications were observed. Three rabbits in each group were sacrificed for analysis. At 4 and 8 weeks, $\mathrm{X}$-rays indicated that bone 

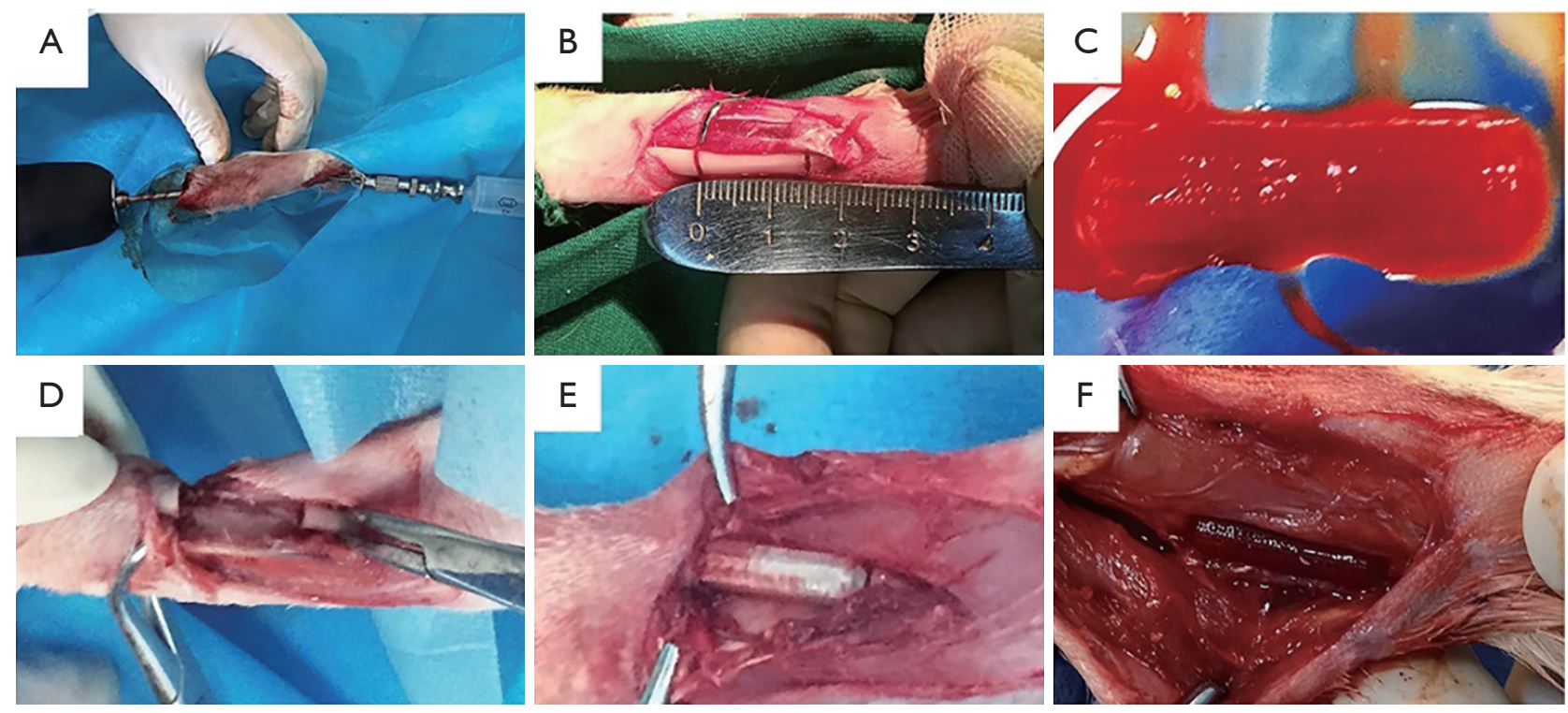

Figure 3 Surgical procedure. (A) The isolation of eBM. (B) The radial bone defect was constructed. (C) eBM was loaded into3D printed PLA-HA scaffolds. (D) The defects were treated with nothing (control group). (E) The defects were treated with 3D printed PLA-HA scaffolds (PLA-HA group). (F) The defects were treated with eBM in conjunction with 3D printed PLA-HA scaffolds (PLA-HA/eBM group).

healing was obviously better in the PLA-HA/eBM group than in the other 2 groups (Figure $4 A$ ). Furthermore, the lane-sandhu $\mathrm{X}$-ray score in the PLA-HA/eBM group was significantly higher than that in the PLA-HA group $(\mathrm{P}<0.01)$, and the scores of both these groups were superior to that of the control group $(\mathrm{P}<0.01)$ (Figure $4 B)$. In the PLA-HA/eBM group, $\mu \mathrm{CT}$ showed bone tissue regeneration to be enhanced compared to that in the PLAHA group (Figure $5 A$ ). At 8 weeks, $\mu \mathrm{CT}$ analysis showed that the BV (Figure 5B) and BV/TV (Figure 5C) in the PLA$\mathrm{HA} / \mathrm{eBM}$ group were significantly higher than those in the PLA-HA group $(\mathrm{P}<0.01)$, and the results of both groups were superior to those of the control group $(\mathrm{P}<0.01)$.

\section{Histological evaluation}

At 8 weeks, a large amount of fibrous tissue and a small amount of new bone had grown at bone defect sites in the control group. The largest amount of new bone tissue formed was observed in the PLA-HA/eBM group (Figure 6A). HE staining indicated that the area of new bone in the PLA-HA/ eBM group was significantly larger than that in the PLA-HA group $(\mathrm{P}<0.01)$, and the results of both groups were superior to those of the control group $(\mathrm{P}<0.01)$ (Figure $6 B)$.

\section{Discussion}

The management of critical-sized bone defects remains a huge challenge, and substitute materials for implantation are needed (27). Currently, numerous strategies have been developed for the treatment of critical-sized bone defects, such as autologous bone transplantation and the application of allografts (28-30). However, as mentioned above, the limitations of these strategies have hampered their application. Over the past decade, eBM tissue has been investigated as a bone graft to facilitate bone repair and regeneration. By providing a large quantity of tissue to fill defect sites, eBM can successfully facilitate bone regeneration. In recent years, BTE has emerged as a new solution for constructing the substitute material for bone repair (31). Researchers have afforded 3D-printing techniques considerable attention for the preparation of advanced BTE scaffolds with biomimetic structures (32). In the present study, we investigated the effect of eBM in conjunction with 3D PLA-HA scaffolds in the repair of long radial defects in rabbits.

Previous studies have indicated that $\mathrm{eBM}$ contains a large quantity of tissue, including fragments of fat, cartilage, bone, and intact pieces of vasculature, and provides concentrations 

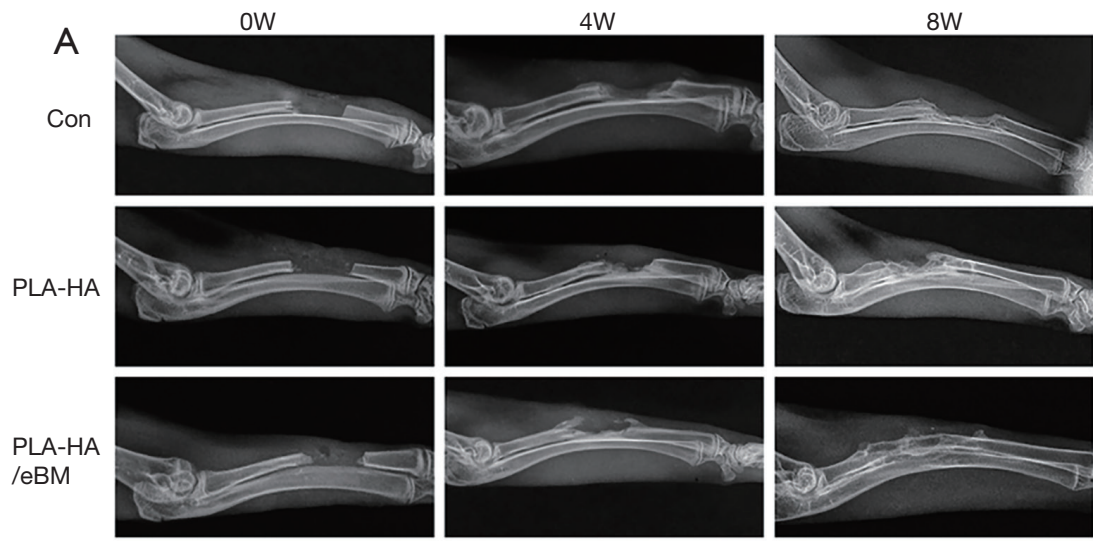

\section{B}

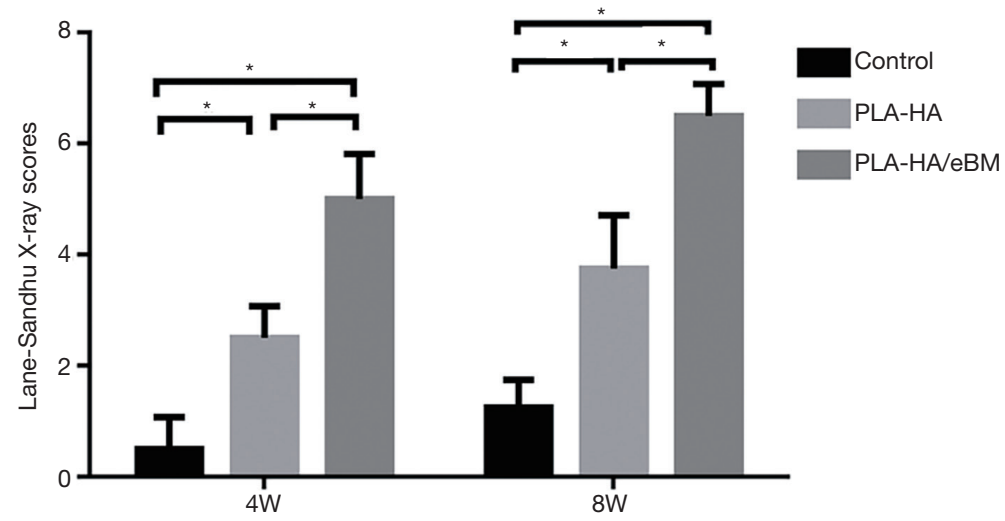

Figure 4 Evaluation of critical-sized bone defect repair with X-rays. (A) X-rays of radial bone defect in control group, PLA-HA group, PLA-HA/eBM group at 0, 4 and 8 weeks. (B) Lane-Sandhu X-ray scores in all groups taken at 4 and 8 weeks post-operatively. ${ }^{*} \mathrm{P}<0.01$.

of osteoprogenitor cells and growth factors that have the potential to facilitate bone repair and regeneration (7). The reamer-irrigator aspirator (RIA) technique can be used to obtain a voluminous amount of bone graft, which appears to have superior growth potential compared to the iliac crest bone graft $(33,34)$. In the present study, we used a 2-mm drill to make holes and ream the distal femur, and a medullo-puncture needle was used in the proximal femur. We successfully obtained large amounts of eBM. Owing to its capacity to accelerate bone repair and regeneration, eBM can serve as a bone substitute graft. However, due to its putty-like shape, eBM cannot impart load-bearing mechanical integrity. In the field of BTE, 3D-printing is a promising technology, as it permits the creation of patientspecific implants with complex porous architectures.

In the present study, 3D-printed PLA-HA scaffolds were successfully fabricated using a $3 \mathrm{D}$-printing technique to provide mechanical support for seeding eBM. Pore size is a scalar quantity that describes the diameter of a hole. The porosity of a material is the percentage of the pore volume of a block material relative to the total volume of the material in its natural state. To facilitate bone formation, bioactive scaffolds should have a porous structure and porosity which are conducive to bone repair, vascular access, and adequate oxygen supply (16). Large pores of 200 $-500 \mu \mathrm{m}$ in the scaffold improve bone growth and capillary formation (35). Similar to long bones, 3D-printed PLA-HA scaffolds are hollow, cylindrical structures. These scaffolds possess a porous wall structure, a pore size of $500 \pm 47.1 \mu \mathrm{m}$, and $60 \% \pm 3.5 \%$ porosity. They can provide adequate room for eBM and an attachment surface onto which MSCs can proliferate and differentiate. Good biocompatibility is an essential property of scaffold materials. Consistent with the findings of previous research (19), we found that MSCs grew well on the surface of PLA-HA scaffolds.

In the current study, critical-sized bone defects were successfully developed in a rabbit model. We harvested eBM using our method, and 3D-printed PLA-HA scaffolds 

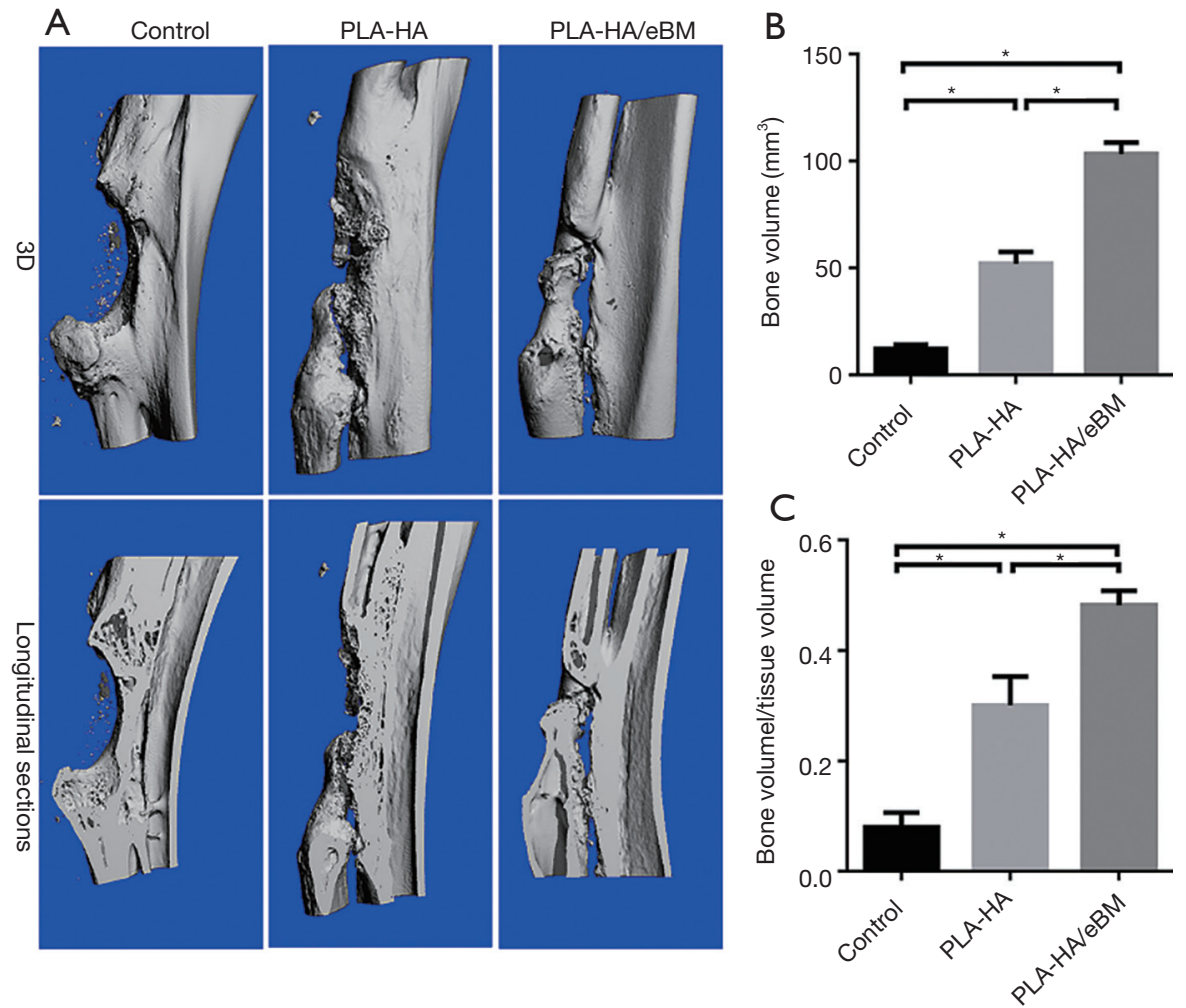

Figure 5 Evaluation of critical-sized bone defect repair with Micro-CT. (A) Representative Micro-CT images (3D and longitudinal sections) of radial long bone defects reconstructed in the control group, the PLA-HA group, and the PLA-HA/eBM group at 8 weeks. (B) bone volume (BV), (C) bone volume/tissue volume (BV/TV) within the defects of the radius at 8 weeks. ${ }^{*} \mathrm{P}<0.01$.
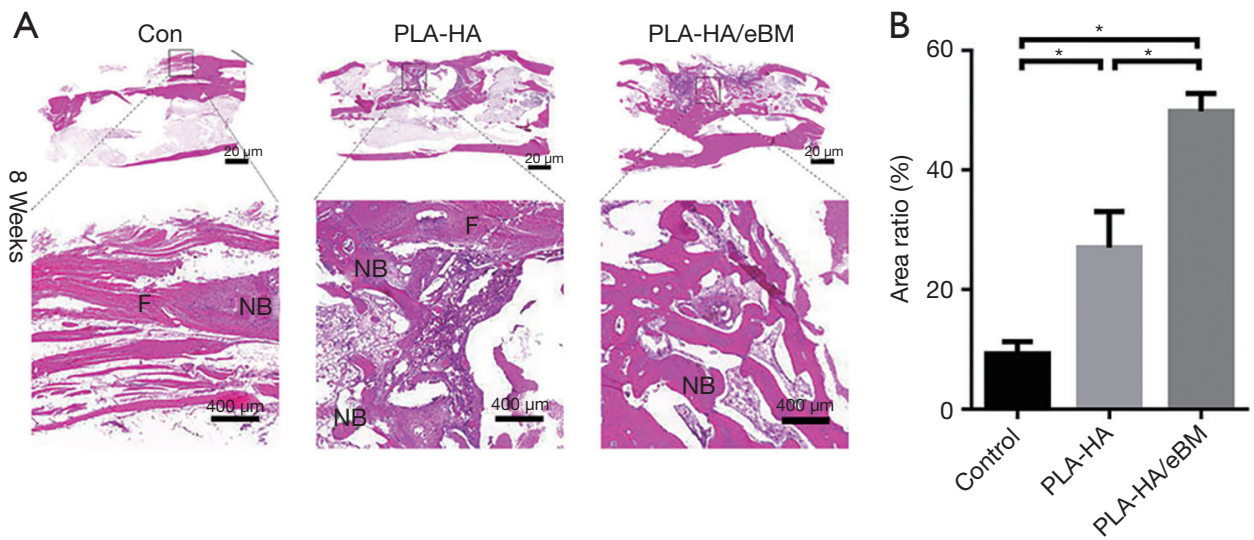

Figure 6 Evaluation of critical-sized bone defect repair with HE staining. (A) Representative images of HE staining in different groups at 8 weeks (NB: new bone; F: fibrous tissue; scale bars $=400 \mu \mathrm{m}$ ). (B) The area ratio of NB at 8 weeks. ${ }^{*} \mathrm{P}<0.01$.

(produced using a 3D-printing technique) were used. Radiological and histological methods were applied to investigate the effect of bone repair and bone regeneration in each group of rabbits. At 4 and 8 weeks, X-rays indicated that bone healing in the PLA-HA/eBM group was obviously better than that in the other 2 groups. Additionally, the lane-sandhu X-ray score in the PLA-HA/eBM group was significantly higher than that in both the PLA-HA group 
and the control group $(\mathrm{P}<0.01)$. At 8 weeks, the $\mu \mathrm{CT}$ analysis showed that BV/TV in the PLA-HA/eBM group was significantly higher than that in the PLA-HA group and the control group $(\mathrm{P}<0.01)$. HE staining indicated that the new bone area in the PLA-HA/eBM group was significantly larger than those in the PLA-HA group and the control group $(\mathrm{P}<0.01)$. The above means of detection also indicated that the treatment in the PLA-HA group was superior to that in the control group. These results suggested that the scaffolds had a number of benefits in relation to the minimization of soft tissue and new bone formation. Further, the results showed that eBM significantly enhanced bone repair and bone regeneration. The results of the $\mathrm{X}$-ray imaging, $\mu \mathrm{CT}$, and histological evaluations suggested that the combination of eBM with PLA-HA achieves a satisfactory bone-healing effect. This new $3 \mathrm{D}$-composite scaffold may be a good material to address the autologous transplantation shortage. Other species, such as sheep and dogs, will be used in further studies.

This study has some limitations. First, due to availability issues, we did not use RIA to acquire eBM. The method we used may have caused more trauma to the animals than RIA would have; however, no animal used in this study became infected or died, which indicates that the method we used was safe. Second, the exploration of 3D-printed PLA-HA was minimal, as the primary purpose of this study was to develop a novel scaffold to facilitate bone formation. Our data were limited, and while this did not affect our research results, further evaluations, including additional qualitative and quantitative studies, may clarify whether this novel scaffold can serve as a bone substitute.

\section{Conclusions}

In the present study, we used eBM in conjunction with 3D-printed PLA-HA scaffolds to repair critical-sized bone defects in a rabbit model. Radiological, $\mu \mathrm{CT}$, and histological techniques were applied to investigate bone regeneration efficacy. The radiological, $\mu \mathrm{CT}$, and histological results revealed that eBM enhances the capacity of PLA-HA for bone repair and regeneration. Thus, the use of eBM in conjunction with 3D PLA-HA scaffolds represents a promising treatment for critical-sized bone defects.

\section{Acknowledgments}

Funding: This work was supported by grants from the
Natural Science Foundation of Shanghai (Grant No. 18ZR1422000), and the National Natural Science Foundation of China (Grant No. 81171729).

\section{Footnote}

Reporting Checklist: The authors have completed the ARRIVE reporting checklist. Available at https://dx.doi. org/10.21037/atm-20-8198

Data Sharing Statement: Available at https://dx.doi. org/10.21037/atm-20-8198

Peer Review File: Available at https://dx.doi.org/10.21037/ atm-20-8198

Conflicts of Interest: All authors have completed the ICMJE uniform disclosure form (available at https://dx.doi. org/10.21037/atm-20-8198). The authors have no conflicts of interest to declare.

Ethical Statement: The authors are accountable for all aspects of the work in ensuring that questions related to the accuracy or integrity of any part of the work are appropriately investigated and resolved. All experiments were performed under a project license (no. HKDL [2018] 499) granted by the Animal Care Committee of Shanghai Jiao Tong University, in compliance with the guidelines for the care and use of animals of Shanghai Ninth People's Hospital Affiliated Shanghai Jiao Tong University School of Medicine.

Open Access Statement: This is an Open Access article distributed in accordance with the Creative Commons Attribution-NonCommercial-NoDerivs 4.0 International License (CC BY-NC-ND 4.0), which permits the noncommercial replication and distribution of the article with the strict proviso that no changes or edits are made and the original work is properly cited (including links to both the formal publication through the relevant DOI and the license). See: https://creativecommons.org/licenses/by-nc-nd/4.0/.

\section{References}

1. Kitasato S, Tanaka T, Chazono M, et al. Local application of alendronate controls bone formation and betatricalcium phosphate resorption induced by recombinant human bone morphogenetic protein-2. J Biomed Mater 
Res A 2020;108:528-36.

2. Liu Z, Ge Y, Zhang L, et al. The effect of induced membranes combined with enhanced bone marrow and $3 \mathrm{D}$ PLA-HA on repairing long bone defects in vivo. $\mathrm{J}$ Tissue Eng Regen Med 2020;14:1403-14.

3. Kuttappan S, Jo JI, Menon D, et al. ONO-1301 loaded nanocomposite scaffolds modulate cAMP mediated signaling and induce new bone formation in critical sized bone defect. Biomater Sci 2020;8:884-96.

4. Singh BN, Veeresh V, Mallick SP, et al. Generation of scaffold incorporated with nanobioglass encapsulated in chitosan/chondroitin sulfate complex for bone tissue engineering. Int J Biol Macromol 2020;153:1-16.

5. Aleczandria S. Tiffany DLG, Toby J. Woods, Kiran Subedi, Brendan A.C. Harley The inclusion of zinc into mineralized collagen scaffolds for craniofacial bone repair applications. Acta Biomaterialia 2019;93:86-96.

6. Bose S, Banerjee D, Robertson S, et al. Enhanced In Vivo Bone and Blood Vessel Formation by Iron Oxide and Silica Doped 3D Printed Tricalcium Phosphate Scaffolds. Ann Biomed Eng 2018;46:1241-53.

7. Hung BP, Salter EK, Temple J, et al. Engineering bone grafts with enhanced bone marrow and native scaffolds. Cells Tissues Organs 2013;198:87-98.

8. Porter RM, Liu F, Pilapil C, et al. Osteogenic potential of reamer irrigator aspirator (RIA) aspirate collected from patients undergoing hip arthroplasty. J Orthop Res 2009;27:42-9.

9. Wenisch S, Trinkaus K, Hild A, et al. Human reaming debris: a source of multipotent stem cells. Bone 2005;36:74-83.

10. Kobbe P, Tarkin IS, Pape HC. Use of the 'reamer irrigator aspirator' system for non-infected tibial non-union after failed iliac crest grafting. Injury 2008;39:796-800.

11. Yan S, Xia P, Xu S, et al. Nanocomposite Porous Microcarriers Based on Strontium-Substituted HAg-Poly( $\gamma$-benzyl-l-glutamate) for Bone Tissue Engineering. ACS Appl Mater Interfaces 2018;10:16270-81.

12. Walsh DP, Raftery RM, Chen G, et al. Rapid healing of a critical-sized bone defect using a collagen-hydroxyapatite scaffold to facilitate low dose, combinatorial growth factor delivery. J Tissue Eng Regen Med 2019;13:1843-53.

13. Zeng H, Pathak JL, Shi Y, et al. Indirect selective laser sintering-printed microporous biphasic calcium phosphate scaffold promotes endogenous bone regeneration via activation of ERK1/2 signaling. Biofabrication 2020;12:025032.

14. Chu L, Jiang G, Hu XL, et al. Osteogenesis, vascularization and osseointegration of a bioactive multiphase macroporous scaffold in the treatment of large bone defects. J Mater Chem B 2018;6:4197-204.

15. Bu S, Yan S, Wang R, et al. In Situ Precipitation of Cluster and Acicular Hydroxyapatite onto Porous Poly(gamma-benzyl-l-glutamate) Microcarriers for Bone Tissue Engineering. ACS Appl Mater Interfaces 2020;12:12468-77.

16. Chu L, Jiang G, Hu XL, et al. Biodegradable macroporous scaffold with nano-crystal surface microstructure for highly effective osteogenesis and vascularization. J Mater Chem B 2018;6:1658-67.

17. Culverwell E, Wimbush S, Hall S. Biotemplated synthesis of an ordered macroporous superconductor with high critical current density using a cuttlebone template. Chem Commun (Camb) 2008;(9):1055-7.

18. Wu D, Spanou A, Diez-Escudero A, et al. 3D-printed PLA/HA composite structures as synthetic trabecular bone: A feasibility study using fused deposition modeling. J Mech Behav Biomed Mater 2020;103:103608.

19. Zhang H, Mao X, Du Z, et al. Three dimensional printed macroporous polylactic acid/hydroxyapatite composite scaffolds for promoting bone formation in a criticalsize rat calvarial defect model. Sci Technol Adv Mater 2016;17:136-48.

20. Senatov FS, Niaza KV, Zadorozhnyy MY, et al. Mechanical properties and shape memory effect of 3D-printed PLAbased porous scaffolds. J Mech Behav Biomed Mater 2016;57:139-48.

21. Sun L, Danoux CB, Wang Q, et al. Independent effects of the chemical and microstructural surface properties of polymer/ceramic composites on proliferation and osteogenic differentiation of human MSCs. Acta Biomater 2016;42:364-77.

22. Persson M, Lorite GS, Kokkonen HE, et al. Effect of bioactive extruded PLA/HA composite films on focal adhesion formation of preosteoblastic cells. Colloids Surf B Biointerfaces 2014;121:409-16.

23. Hassanajili S, Karami-Pour A, Oryan A, et al. Preparation and characterization of PLA/PCL/HA composite scaffolds using indirect 3D printing for bone tissue engineering. Mater Sci Eng C Mater Biol Appl 2019;104:109960.

24. Fu S, Liu $W$, Liu $S$, et al. $3 \mathrm{D}$ printed porous $\beta$-Ca2SiO4 scaffolds derived from preceramic resin and their physicochemical and biological properties. Sci Technol Adv Mater 2018;19:495-506.

25. Green J. History and development of suction-irrigationreaming. Injury 2010;41:S24-S31. 
26. Ghanmi S, Trigui $M$, Baya $W$, et al. The periosteumlike effect of fresh human amniotic membrane on bone regeneration in a rabbit critical-sized defect model. Bone 2018;110:392-404.

27. Omata K, Matsuno T, Asano K, et al. Enhanced bone regeneration by gelatin- $\beta$-tricalcium phosphate composites enabling controlled release of bFGF. J Tissue Eng Regen Med 2014;8:604-11.

28. Cui Y, Zhu T, Li A, et al. Porous Particle-Reinforced Bioactive Gelatin Scaffold for Large Segmental Bone Defect Repairing. ACS Appl Mater Interfaces 2018;10:6956-64.

29. Dasgupta S, Maji K, Nandi SK. Investigating the mechanical, physiochemical and osteogenic properties in gelatin-chitosan-bioactive nanoceramic composite scaffolds for bone tissue regeneration: In vitro and in vivo. Mater Sci Eng C Mater Biol Appl 2019;94:713-28.

30. Jiang F, Yin F, Lin Y, et al. The promotion of bone regeneration through CS/GP-CTH/antagomir-133a/b

Cite this article as: Liu Z, Chu W, Zhang L, Wang Y, Zhai Z, Liu F. The effect of enhanced bone marrow in conjunction with 3D-printed PLA-HA in the repair of critical-sized bone defects in a rabbit model. Ann Transl Med 2021;9(14):1134. doi: 10.21037/ atm-20-8198 sustained release system. Nanomedicine 2020;24:102116.

31. Söhling N, Neijhoft J, Nienhaus V, et al. 3D-Printing of Hierarchically Designed and Osteoconductive Bone Tissue Engineering Scaffolds. Materials (Basel) 2020;13:1836.

32. Cakmak AM, Unal S, Sahin A, et al. 3D Printed Polycaprolactone/Gelatin/Bacterial Cellulose/ Hydroxyapatite Composite Scaffold for Bone Tissue Engineering. Polymers (Basel) 2020;12:1962.

33. van der Bel R, Blokhuis TJ. Increased osteogenic capacity of Reamer/Irrigator/Aspirator derived mesenchymal stem cells. Injury 2014;45:2060-4.

34. Henrich D, Seebach C, Sterlepper E, et al. RIA reamings and hip aspirate: A comparative evaluation of osteoprogenitor and endothelial progenitor cells. Injury 2010;41:S62-8.

35. Zhang Y, Xia L, Zhai D, et al. Mesoporous bioactive glass nanolayer-functionalized 3D-printed scaffolds for accelerating osteogenesis and angiogenesis. Nanoscale 2015;7:19207-21. 